\title{
Nanolipoprotein mediated Her2 protein transfection induces malignant transformation in human breast acinar cultures
}

\author{
Wei He ${ }^{2}$, Angela C. Evans ${ }^{3}$, Matthew Coleman ${ }^{2,3}$, Claire Robertson ${ }^{1}$ \\ 1: Materials Engineering Division, Lawrence Livermore National Lab. 7000 East Ave, Livermore, CA, 94550, USA \\ 2: Physical and Life Sciences Division, Lawrence Livermore National Lab. 7000 East Ave, Livermore, CA, 94550, USA \\ 3: Department of Radiation Oncology, University of California Davis, School of Medicine, Sacramento, CA, 95817, \\ USA
}

\begin{abstract}
Her2 overexpression is associated with an aggressive form of breast cancer and malignant transformation. We sought to determine if a nano-carrier system could introduce Her 2 protein to non-malignant cells and determine the effects on cell phenotype and transcriptome. With stimulation with Her2-NLPs, we observed uptake of Her2 protein and a decreased probability of individual non-malignant cells forming polar growth arrested acinar-like structures. The NLP delivery system alone or Her2-NLPs plus trastuzumab showed no effect on acinar organization rate. Transcriptomics revealed essentially no effect of empty NLPs versus untreated cells whereas Her2-NLPs versus either untreated or empty NLP treated cells revealed upregulation of several factors associated with breast cancer. Pathway analysis also suggested that known nodes downstream of Her 2 were activated in response to Her2-NLP treatment. This suggests that Her2-NLPs are sufficient for malignant transformation of non-malignant cells and that this system offers a new model for studying cell surface receptor signaling without genomic modification or transformation techniques.
\end{abstract}

\section{Introduction:}

Half of all drugs that have been approved to treat cancer in the past decade target cell surface receptors ${ }^{1}$. Despite the clinical success of these compounds, drug resistance remains an issue: for example, Her2 (Human epidermal growth factor family receptor 2, aka ERBB2) overexpressing breast cancers are treated with Her2 inhibitors such as trastuzumab, pertuzumab, and nivolumab, but $\sim 15 \%$ of Her 2 overexpressing cancers do not respond to these drugs at baseline $e^{2}$ and most patients develop resistance within 1 year ${ }^{3}$.

The signaling downstream of Her 2 which promotes growth and malignancy is a potential source of alternative targets; however, studying this signaling is complicated by the growth advantage provided by Her $2^{4,5}$. Some groups avoid this selection by using artificially dimerizing Her 2 transgene systems ${ }^{6-8}$, but these systems differ significantly in their activation from wildtype Her2. Thus, we sought to determine whether a recently described protein delivery system could transport Her 2 to breast cells and whether this culture system could be used as a new model of Her2-overexpressing breast cancer.

Nanolipoprotein particles (NLPs), comprised of lipid surrounded by apolipoproteins, allow for efficient solubilization of functional membrane embedded proteins ${ }^{9-14}$, and transfer to cells without transgenic modification or artificial cell selection ${ }^{10}$. NLPs, in contrast, to other protein delivery systems, do not require cell membrane disruption ${ }^{15-18}$ and diffuse readily due to their small size relative to silica or PLGA nanoparticles ${ }^{10,13,19-}$ ${ }^{21}$. Furthermore, NLPs maintain correct folding of mammalian cell surface receptors ${ }^{11,12,14}$ whereas many delivery systems risk denaturing the proteins they carry, either by harsh packaging or endosomal lytic processes ${ }^{18,22,23}$. Importantly, Her 2 supported NLPs retain tyrosine kinase functionality and sensitivity to Her 2 inhibitors, even when synthesized in prokaryotic lysates ${ }^{13}$. 
We chose to determine the effects of Her2-NLPs in the breast acinar morphogenesis model. Non-malignant cells cultured in 3D laminin-rich gels form growth-arrested acinar-like structures that resemble normal breast, whereas malignant cells form apolar structures growing tumor-like colonies ${ }^{24,25}$. Importantly, signaling downstream of Her2 has been shown to block acinar morphogenesis in similar models ${ }^{6-8,26}$ : if Her 2 transported in NLPs is sufficient for malignant transformation, cells will organize into tumor-like structures instead of acinar-like structures. We found that NLPs readily transported Her2 to non-malignant breast cells, induced tumor-like phenotypic changes and transcriptomic changes.

\section{Results and Discussion:}

\section{Her2-NLPs transfer functional Her2 protein to cells in 3D culture}

Her2-NLPs and control empty NLPs (lipid and apolipoprotein only) were synthesized in a E. coli bacterial cell-free lysate and affinity purified as previously described ${ }^{13}$. We checked for presence of endotoxin and found that Empty NLPs contained an average endotoxin level of $104 \mathrm{EU} / \mathrm{mg}$ total protein and Her2-NLPs contained $160 \mathrm{EU} / \mathrm{mg}$ total protein. When diluted to $5 \mu \mathrm{g} / \mathrm{ml}$ for cell culture, final endotoxin concentrations were less than $1 \mathrm{EU} / \mathrm{ml}$, which represents a commonly used threshold for "endotoxin free" cell culture media. Non-malignant HMT3522-S1 cells in $3 \mathrm{~d}$ culture were then stimulated with $5 \mu \mathrm{g} / \mathrm{ml} \mathrm{Her2-NLP}$ or Empty-NLPs. Non-malignant cells treated with Her2NLPs demonstrated by positive staining for Her 2 at 18 hours of culture (Supplementary Fig. 1).

\section{Her2-NLPs cause non-malignant S1 cells to disorganize into malignant like structures}

To determine the phenotypic consequences of NLP mediated Her2 transfer, we cultured HMT-3522-S1 and -T4-2 cells in 3D laminin rich ECM (IrECM) hydrogels as previously described ${ }^{25}$. Briefly, single cells were dispersed in LrECM and allowed to gel, then overlaid with either culture media, or culture media $+5 \mu \mathrm{g} / \mathrm{ml}$ of Her $2 \mathrm{NLPs}$. Media and NLPs were replaced every 2-3 days for 10 days. Samples were then fixed and stained to manually identify features associated with cellular polarity. Power analysis revealed that for a chi-square test assuming an effect size of 0.33 , and a significance level of 0.05 , scoring 100 structures would give a power of $>0.8$, thus a total of 100 structures per condition per experiment were scored.

As previously reported, the majority of non-malignant $\mathrm{S} 1$ cells (Fig. $1 \mathrm{~A})$ formed growth arrested $(<4 \%$ contained a mitotic figure) polar structures (60\% \pm well organized) whereas almost all malignant T4 (Fig. 1B) cells formed apolar structures, with $30 \%$ of structures displaying 1 or more mitotic figures at the end of culture. Her 2-NLP treated S1 cells (Fig. 1C) were significantly more likely to disorganize and to contain mitotic figures, compared to untreated S1 across 5 independent experiments (Pearson's Chi-square test $p<10^{-14}$ ). To ensure that the observed effects were due to Her2 and not the nanocarrier system, we repeated NLP stimulation experiments with Empty-NLPs (Fig. 1D) comprised of lipid and apolipoprotein alone at $5 \mu \mathrm{g} / \mathrm{ml}$, and with Her2-NLPs + a Her 2 function blocking antibody inhibitor (4C5-8, a trastuzumab biosimilar) at a 1:2 molar ratio (Fig. 1E). Trastuzumab is a monoclonal antibody that is clinically used to treat HER2 positive breast cancer by binding to a conformational epitope of Her2 protein in its extracellular juxtamembrane domain and block its function. In our previous study, we have demonstrated that 4C5-8 binds to cell-free produced Her2-NLP similar to native Her 2 protein ${ }^{13}$, and in the current experiment, we observed no effect of either empty-NLPs or Her2-NLPs in combination with Her2 targeted antibody that blocks receptor function (Fig. 1F). This shows that Her 2 itself drives nonmalignant cells to disorganize and fail to growth arrest, while the supporting NLP system has minimal phenotypic effects. Titrating the Her2-NLP concentration revealed dose response behavior, with increasing probability of disorganization with increasing levels of delivered Her2 (Fig. 1G). Increased numbers of mitotic cells were also observed in Her2-NLP treated structures relative to either non-malignant or empty-NLP treated cells (Fig. 1H).

To the best of our knowledge, this is the first demonstration that protein transfer of an oncoprotein is sufficient to drive malignant transformation of non-malignant breast cells in 3D culture. Akin to previous work using artificially dimerizing $\mathrm{Her}^{7}$, our work demonstrates that Her 2 can block acinar morphogenesis, however, instead of these relatively complex transgenic strategies, we use wildtype Her2 protein. This work is distinct from previous experiments studying the effects of Her2 using genomic modification, as we do not induce genomic breaks, nor 
induce an immune response to cytoplasmic DNA ${ }^{27}$ nor select cells. We did not directly measure dimerization state in these models, however treatment with a Her2 dimerization inhibitor blocked the effect of Her2-NLPs, indicating correct folding and presentation of the receptor.

\section{Her2-NLPs cause transcriptomic changes in non-malignant cells}

To understand the transcriptomic changes induced by increased levels of Her2, we cultured non-malignant $S 1$ cells with no treatment, treated with $5 \mu \mathrm{g} / \mathrm{ml}$ empty-NLP, or treated with $5 \mu \mathrm{g} / \mathrm{ml}$ Her2-NLP in 3D LrECM for 8-10 days. Cells were then extracted from the IrECM hydrogen, then lysed to extract total RNA for 2 biologically independent experiments. The RNA was isolated and enriched through poly-A selection and sequenced using nextgen sequencing (HiSeq, illumina). Reads were normalized, then mapped to the human genome and compared across groups using DESeq2 using a $p$-value cutoff of $<0.05$, and an absolute $\log _{2}$ fold change of $>1$. PCA analysis revealed that replicate was the primary cause of variability between experiments, followed by Her2-NLP treatment (Fig. 2A).

Comparing untreated and empty-NLP treated cells revealed only 1 differentially expressed transcript, the noncoding IncRNA BCYRN1. Gene ontology analysis also revealed no differentially expressed terms, further emphasizing the minimal effects of the NLP system (Fig. 2B and Supplementary Fig. 2). In contrast, comparing empty-NLPs and Her2-NLPs revealed 9 upregulated genes and comparing no treatment and Her2-NLPs revealed 32 upregulated genes and 6 downregulated genes. Four genes were consistently upregulated with Her2-NLP treatment as compared to either empty-NLP alone or untreated cells (Fig. 2B). Transcripts identified in Her2-NLP treated groups relative to either untreated or empty-NLP treated factors overlapped with previously reported screens for acinar morphogenesis $(\mathrm{EBI} 3, \mathrm{CCL} 5)^{28}$ and for residual disease in Her $2+$ breast cancer $\left(\mathrm{CCL} 5^{29,30}\right)$, breast cancer outcome $\left(\mathrm{EBI}^{31-33}\right)$ and metastasis $\left(\mathrm{TGM}^{34}, \mathrm{EBI}^{33,35}\right)$.

Differentially expressed genes comparing Her2-NLP treatment to empty-NLPs or to untreated cell cultures were analyzed via Ingenuity Pathway Analysis (IPA). Her2-NLP treated cells compared to either untreated or empty-NLP treated cells showed a clear activation of cancer-related biological responses (Supplementary Table 2) and predicted activation of signaling nodes which have been linked to Her 2 overexpression such as Stat $3^{36}$, ERK and $\mathrm{TgFB}^{26}$, p38MAPK ${ }^{37}$, and NFkB ${ }^{28}$ (Fig. $2 \mathrm{C}$ and Supplementary Fig. 3). Notably, signatures of cancer-related malignancies, including breast and ovarian cancers, were identified as prominently associated with HER-2 NLP treatment as compared to empty-NLPs (Supplementary Fig 4). The strongest disease networks associated with Her2-NLP treatment were activation of secondary tumor formation and suppression of organ degeneration (Fig. 2D-E). Her2-NLP treatment was also linked with several cancer-related disease behaviors, including proliferation, the synthesis of reactive oxygen species, and formation of cellular protrusions. Upstream factor analysis suggested that common upstream nodes may include NFKB, lipopolysaccharide, interferon gamma, and TNF (Supplementary Table 3).

Our demonstration that Her2-NLPs induce malignant like phenotypic and transcriptomic changes shows that this model represents an oncoprotein driven model of malignant transformation. Specifically, predicted activation of breast cancer associated signaling nodes suggests that our model recapitulates features of Her 2 overexpressing breast cancer and may offer new avenues to study Her 2 signaling in heterogeneous backgrounds. Future work using this system can take advantage of the lack of alternative splicing of Her2, dynamic introduction of Her 2 for pulse-chase experiments, and use the reversible nature of this system to study Her 2 withdrawal.

\section{Conclusions}

In this work, we demonstrate, for the first time to our knowledge, that receptor-driven oncoprotein transfection can induce malignant transformation in phenotypic cultures. Specifically, we demonstrate that the Her 2 receptor transferred to non-malignant breast cells induces malignant like growth patterns in a subset of cells, and that this effect is not seen either with the protein transfer system (NLP alone) or Her 2-NLP in concert with a conformational dimerization inhibitor. This protein delivery system is limited to membrane bound proteins, but given the centrality of the surface receptors in cancer, including the tyrosine kinase receptor family and the G protein- 
coupled receptor family, there is a clear use for this system in rapidly engineering receptor driven cancers, which represent targets for over half of all cancer drugs approved in the last decade.

Acknowledgements:

Funding: Lawrence Livermore National Lab Laboratory Directed Research Program 18-ERD-062 (to CR) and 19-SI003.

Thanks to Christine Ichim, Monica Moya, W. Rick Hynes for their helpful discussion related to this work. Thanks to Aimy Sebastian for help with bioinformatics analysis.

This work was performed under the auspices of the U.S. Department of Energy by Lawrence Livermore National Laboratory under Contract DE-AC52-07NA27344. IM release number LLNL-JRNL-813643 


\section{Supplementary Materials and Methods: NLP Preparation}

Her2-NLPs were prepared as previously described ${ }^{13}$. Briefly, plasmids encoding for human full-length ErbB2 gene and a truncated $6 \mathrm{x}$-His-tagged apolipoprotein A1 ( $\triangle 49 \mathrm{~A} 1)$ were synthesized using the cell-free Expressway system (Life Technologies), in the presence of 1,2-dimyristoyl-sn-glycero-3-phosphocholine (DMPC, Avanti). After overnight expression, Her2-NLPs were harvested from the cell-free mixture by native nickel pulldown ${ }^{13}$. The purified NLP stocks then underwent buffer exchange into pH7.4 PBS and sterile filtration for use in antibiotic free mammalian cell cultures. Empty NLPs were made using same cell-free method except for without ErbB2 plasmid. Protein concentrations were determined by Nandrop. Endotoxin concentrations were determined using the Endosafe?-PTSTM (Charles River) endotoxin testing system based on the Limulus amebocyte lysate assay and is expressed as EU/mg total protein.

\section{Cell Culture}

Human immortalized breast cells (S1) and breast cancer cells (T4-2) from the HMT3522 progression series (kind gift of Mina Bissell) ${ }^{38}$ were maintained in DMEM/F12 (11330, ThermoFisher) supplemented with $250 \mathrm{ng} / \mathrm{ml}$ insulin

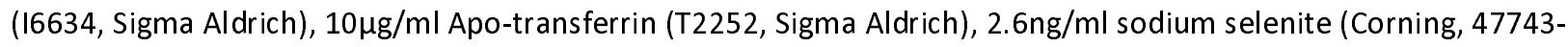
618), $10^{-10} \mathrm{M}$ beta-estradiol (E2785, Sigma Aldrich), 1.4×10-6 M hydrocortisone (H0888, Sigma Aldrich), $5 \mu \mathrm{g} / \mathrm{ml}$ ovine prolactin (LA Biomedical Research Institute), and for S1 only, 10ng/ml EGF (11376454001, Roche). S1 were seeded in uncoated T75 flasks (corning) at $2 \mathrm{e}^{4} / \mathrm{cm}^{2}$, refed every 2 days, and passed every 7 days and T4-2 were seeded in collagen coated flasks (corning) at $1 \mathrm{e}^{4} / \mathrm{cm}^{2}$, refed every 2 days and passed every 5 and 3 days respectively. Cells were kept in humidified incubators at $37 \mathrm{C}$ and $5 \% \mathrm{CO} 2$ supplementation, and $\mathrm{CO} 2$ calibrations were performed biweekly with a test kit (Fyrite, Bacharach). Cells were screened for mycoplasma contamination every 2 months (Mycoalert, Lonza).

\section{Acinar Morphogenesis Assay}

S1 or T4-2 cells were passaged, counted, and resuspended in 100\% IrECM (354230, growth factor reduced Matrigel, Corning) at $800 \mathrm{k}$ cell $\mathrm{s} / \mathrm{ml}$ or $600 \mathrm{k} / \mathrm{ml}$ respectively on ice. Cells in IrECM were transferred to dishes, allowed to gel for 20 minutes at $37 \mathrm{C}$, then covered with complete culture media $\pm 5 \mu \mathrm{g} / \mathrm{ml}$ Her 2-NLPs or empty NLPs or Her2-NLPs plus a Her2 blocking antibody (clone 4D5, MCA6092, Biorad) at $26.67 \mu \mathrm{g} / \mathrm{ml}$ (which represented 1:1 molar ratio with Her2-NLPS). Media was replaced every 2-3 days for 10 days.

At 10 days, cultures were harvested for imaging by removing culture media and smearing cell laden IrECM onto coated glass slides (Superfrost plus, Thermo). Slides were then immediately fixed in $10 \%$ formalin for 15 minutes, washed $3 x$ in PBS, blocked in for $1 \mathrm{hr}$ at room temp in IF buffer comprised of $3 \%$ BSA and $0.5 \%$ Triton X-100 in PBS supplemented with $10 \%$ normal goat serum and goat anti-mouse Fab fragment (115-007-003, Jackson Immunoresearch) to block mouse antibodies present in IrECM. Primary antibodies for beta-catenin (Ab32572 Abcam), alpha-6 integrin (555734, BD Biosciences) and laminin (L9393, Millipore Sigma) were diluted 1:100 in IF buffer and stained for $2 \mathrm{hrs}$ at room temperature. Slides were then washed $3 \mathrm{x}$ in IF buffer, and secondary antibodies (A21429, A-11006, A32723 as appropriate, Thermo Fisher) were applied at 1:500 dilution in IF buffer for $1 \mathrm{hr}$ at room temp followed by 3 washes with IF buffer, and 3 washes with PBS. Slides were then stained with dapi at $0.1 \mu \mathrm{g} / \mathrm{ml}$ for 5 minutes and mounted (Prolong Gold, Thermo Fisher) and cover slipped.

Slides were imaged on a laser scanning confocal microscope (LSM700, Zeiss) equipped with an Acroplan 40x/1.1NA water immersion lens. 100 structures per experiment were scored by a trained observer according to criteria in Supplementary Table 1. Pearson's Chi-square test and Bonferroni post hoc tests were performed in R.

\section{RNAseq}

After 10 days of culture, cells were harvested from IrECM by incubation with 5mM EDTA in PBS on ice for 30 minutes with gentle agitation and spun down to collect structures. Cell pellets were then snap frozen on dry ice for shipping, followed by RNA extraction, poly-A capture, CDNA synthesis, end repair and adaptor ligation. Samples 
were then sequenced (HiSeq, Illumina). Reads were trimmed to remove adapter sequences and poor-quality nucleotides, then aligned to the Homo Sapiens reference genome (GRCh38). Differentially expressed genes were compared using DEGseq2. The Wald test was used to generate $p$-values and log2 fold changes. Genes with an unadjusted $p$-value $<0.05$ and absolute log 2 fold change $>1$ were called as differentially expressed genes for each comparison. A gene ontology analysis was performed on the statistically significant set of genes by implementing the software GeneSCF v.1.1-p2. The goa_human GO list was used to cluster the set of genes based on their biological processes and determine their statistical significance. To estimate the expression levels of alternatively spliced transcripts, the splice variant hit counts were extracted from the RNA-seq reads mapped to the genome. Differentially spliced genes were identified for groups with more than one sample by testing for significant differences in read counts on exons (and junctions) of the genes using DEXSeq.

Ingenuity Pathway Analysis (IPA) Differentially expressed genes from RNAseq studies were run through IPA (Qiagen). In total, three core analyses were run; Empty-NLP vs. No treatment, Her2-NLP vs. No treatment, and Her2-NLP vs. empty-NLP. Both direct and indirect relationships were considered for IPA mapping and statistical analysis. $\log _{2}$ fold change cutoffs of $+/-1$ and $p$-values $<0.05$ were included in the analysis. 


\section{References}

1 DiMasi, J. A., Reichert, J. M., Feldman, L. \& Malins, A. Clinical Approval Success Rates for Investigational Cancer Drugs. Clinical Pharmacology \& Therapeutics 94, 329-335, doi:10.1038/clpt.2013.117 (2013).

2 Daniels, B. et al. Long-term survival in trastuzumab-treated patients with HER2-positive metastatic breast cancer: real-world outcomes and treatment patterns in a whole-of-population Australian cohort (20012016). Breast Cancer Research and Treatment 171, 151-159, doi:10.1007/s10549-018-4804-0 (2018).

3 Cobleigh, M. A. et al. Multinational study of the efficacy and safety of humanized anti-HER2 monoclonal antibody in women who have HER2-overexpressing metastatic breast cancer that has progressed after chemotherapy for metastatic disease. Journal of clinical oncology : official journal of the American Society of Clinical Oncology 17, 2639-2648, doi:10.1200/jco.1999.17.9.2639 (1999).

4 Ben-David, U. et al. Genetic and transcriptional evolution alters cancer cell line drug response. Nature 560, 325-330, doi:10.1038/s41586-018-0409-3 (2018).

5 Stepanenko, A. A. \& Heng, H. H. Transient and stable vector transfection: Pitfalls, off-target effects, artifacts. Mutation research 773, 91-103, doi:10.1016/j.mrrev.2017.05.002 (2017).

6 Zhan, L., Xiang, B. \& Muthuswamy, S. K. Controlled Activation of ErbB1/ErbB2 Heterodimers Promote Invasion of Three-Dimensional Organized Epithelia in an ErbB1-Dependent Manner: Implications for Progression of ErbB2-Overexpressing Tumors. Cancer Research 66, 5201-5208, doi:10.1158/00085472.can-05-4081 (2006).

7 Muthuswamy, S. K., Li, D., Lelievre, S., Bissell, M. J. \& Brugge, J. S. ErbB2, but not ErbB1, reinitiates proliferation and induces luminal repopulation in epithelial acini. Nature Cell Biology 3, 785, doi:10.1038/ncb0901-785

https://www.nature.com/articles/ncb0901-785\#supplementary-information (2001).

8 Muthuswamy, S. K., Gilman, M. \& Brugge, J. S. Controlled dimerization of ErbB receptors provides evidence for differential signaling by homo- and heterodimers. Molecular and cellular biology 19, 68456857, doi:10.1128/mcb.19.10.6845 (1999).

$9 \quad$ Katzen, F. et al. Insertion of Membrane Proteins into Discoidal Membranes Using a Cell-Free Protein Expression Approach. Journal of Proteome Research 7, 3535-3542, doi:10.1021/pr800265f (2008).

10 Patriarchi, T. et al. Nanodelivery of a functional membrane receptor to manipulate cellular phenotype. Scientific Reports 8, 3556, doi:10.1038/s41598-018-21863-3 (2018).

11 Gao, T. et al. Characterizing diffusion dynamics of a membrane protein associated with nanolipoproteins using fluorescence correlation spectroscopy. Protein Science 20, 437-447, doi:10.1002/pro.577 (2011).

12 Shelby, M. L., He, W., Dang, A. T., Kuhl, T. L. \& Coleman, M. A. Cell-Free Co-Translational Approaches for Producing Mammalian Receptors: Expanding the Cell-Free Expression Toolbox Using Nanolipoproteins. Front Pharmacol 10, 744-744, doi:10.3389/fphar.2019.00744 (2019).

13 He, W. et al. Cell-free expression of functional receptor tyrosine kinases. Sci Rep 5, 12896, doi:10.1038/srep12896 (2015).

14 Cappuccio, J. A. et al. Cell-free Co-expression of Functional Membrane Proteins and Apolipoprotein, Forming Soluble Nanolipoprotein Particles. Molecular \&amp; Cellular Proteomics 7, 2246-2253, doi:10.1074/mcp.M800191-MCP200 (2008).

15 Takeuchi, T. et al. Direct and Rapid Cytosolic Delivery Using Cell-Penetrating Peptides Mediated by Pyrenebutyrate. ACS Chemical Biology 1, 299-303, doi:10.1021/cb600127m (2006).

16 Stewart, M. P., Langer, R. \& Jensen, K. F. Intracellular Delivery by Membrane Disruption: Mechanisms, Strategies, and Concepts. Chemical Reviews 118, 7409-7531, doi:10.1021/acs.chemrev.7b00678 (2018). Xiong, R. et al. Cytosolic Delivery of Nanolabels Prevents Their Asymmetric Inheritance and Enables Extended Quantitative in Vivo Cell Imaging. Nano Letters 16, 5975-5986, doi:10.1021/acs.nanolett.6b01411 (2016). Kaplan, I. M., Wadia, J. S. \& Dowdy, S. F. Cationic TAT peptide transduction domain enters cells by macropinocytosis. Journal of Controlled Release 102, 247-253, doi:https://doi.org/10.1016/i.jconrel.2004.10.018 (2005). Advanced Drug Delivery Reviews 55, 329-347, doi:https://doi.org/10.1016/S0169-409X(02)00228-4 (2003). 
Leifert, J. A., Harkins, S. \& Whitton, J. L. Full-length proteins attached to the HIV tat protein transduction domain are neither transduced between cells, nor exhibit enhanced immunogenicity. Gene therapy 9 , 1422-1428, doi:10.1038/sj.gt.3301819 (2002). targeting and visualization in real time. Scientific Reports 6, 25019, doi:10.1038/srep25019 (2016). Erazo-Oliveras, A. et al. Protein delivery into live cells by incubation with an endosomolytic agent. Nature Methods 11, 861-867, doi:10.1038/nmeth.2998 (2014).

23 Lee, Y.-J., Johnson, G., Peltier, G. C. \& Pellois, J.-P. A HA2-Fusion tag limits the endosomal release of its protein cargo despite causing endosomal lysis. Biochimica et biophysica acta 1810, 752-758, doi:10.1016/j.bbagen.2011.05.013 (2011).

24 Weaver, V. M. et al. Reversion of the malignant phenotype of human breast cells in three-dimensional culture and in vivo by integrin blocking antibodies. The Journal of cell biology 137, 231-245 (1997). Lee, G. Y., Kenny, P. A., Lee, E. H. \& Bissell, M. J. Three-dimensional culture models of normal and malignant breast epithelial cells. Nature Methods 4, 359, doi:10.1038/nmeth1015

https://www.nature.com/articles/nmeth1015\#supplementary-information (2007).

26 Seton-Rogers, S. E. et al. Cooperation of the ErbB2 receptor and transforming growth factor $\beta$ in induction of migration and invasion in mammary epithelial cells. Proceedings of the National Academy of Sciences of the United States of America 101, 1257-1262, doi:10.1073/pnas.0308090100 (2004). Ishikawa, H., Ma, Z. \& Barber, G. N. STING regulates intracellular DNA-mediated, type I interferondependent innate immunity. Nature 461, 788-792, doi:10.1038/nature08476 (2009). Becker-Weimann, S. et al. NFkB disrupts tissue polarity in 3D by preventing integration of microenvironmental signals. Oncotarget 4, 2010-2020, doi:10.18632/oncotarget.1451 (2013). Denkert, C. et al. Expression of immunologic genes in triple-negative and HER2-positive breast cancer in the neoadjuvant GEPARSIXTO trial: Prediction of response to carboplatin-based chemotherapy. Journal of Clinical Oncology 32, 510-510, doi:10.1200/jco.2014.32.15_suppl.510 (2014). Zazo, S. et al. Autocrine CCL5 Effect Mediates Trastuzumab Resistance by ERK Pathway Activation in HER2Positive Breast Cancer. Molecular Cancer Therapeutics 19, 1696-1707, doi:10.1158/1535-7163.mct-191172 (2020). Jiang, J. \& Liu, X. Upregulated EBI3 Correlates with Poor Outcome and Tumor Progression in Breast Cancer. Oncology Research and Treatment 41, 111-115, doi:10.1159/000484935 (2018). Chen, G. et al. Circulating low IL-23: IL-35 cytokine ratio promotes progression associated with poor prognosisin breast cancer. Am J Trans/ Res 8, 2255-2264 (2016).

33 Jiang, J. \& Liu, X. Upregulated EBI3 Correlates with Poor Outcome and Tumor Progression in Breast Cancer. Oncol Res Treat 41, 111-115, doi:10.1159/000484935 (2018). Shinde, A. et al. Transglutaminase- 2 facilitates extracellular vesicle-mediated establishment of the metastatic niche. Oncogenesis 9, 16, doi:10.1038/s41389-020-0204-5 (2020). Liang, Y. et al. Epstein-Barr Virus-Induced Gene 3 (EBI3) Blocking Leads to Induce Antitumor Cytotoxic T Lymphocyte Response and Suppress Tumor Growth in Colorectal Cancer by Bidirectional ReciprocalRegulation STAT3 Signaling Pathway. Mediators of Inflammation 2016, 3214105, doi:10.1155/2016/3214105 (2016). Guo, W. et al. $\beta 4$ Integrin Amplifies ErbB2 Signaling to Promote Mammary Tumorigenesis. Cell 126, 489502, doi:https://doi.org/10.1016/i.cell.2006.05.047 (2006). Woods Ignatoski, K. M., Grewal, N. K., Markwart, S., Livant, D. L. \& Ethier, S. P. p38MAPK induces cell surface alpha4 integrin downregulation to facilitate erbB-2-mediated invasion. Neoplasia 5, 128-134, doi:10.1016/s1476-5586(03)80004-0 (2003). luminal breast epithelial cells for polarity and basement membrane deposition. J Cell Sci 115, 39-50 (2002). 


\section{Figures}

Figure 1: Her2 carried by NLPs causes malignant-like organization in non-malignant cells. A: Non-malignant cells cultured in IrECM form growth arrested polarized acinar like structure with basal organization of $\alpha 6$ integrin (green), and lateral organization of $\beta$-catenin (red). B: Malignant breast cancer cells form disorganized structures with no cell polarity which fail to growth arrest as shown by presence of mitotic figures (white arrow). C: Nonmalignant cells treated with Her2-NLPs form apolar masses which fail to growth arrest. D: In contrast, nonmalignant cells treated with empty-NLPs organize normally. E: non-malignant cells treated Her2-NLPs and a Her2 dimerization inhibitory antibody organize normally. F: Significantly more structures organize well in Nonmalignant, empty-NLP treated, inhibitory antibody only and Her2-NLP+ inhibitory antibody conditions, whereas Her2-NLP treated and malignant cells are less likely to organize into polarized, growth arrested acini ( $n=5$ biological replicates, * indicates post hoc test significance of $p<0.05, * *$ indicates post hoc test of $p<0.001)$. F: Her $2-N L P s$ show dose response behavior, with fewer structures organizing well with increasing NLP dosage ( $n=3$ biological replicates, * indicates post hoc test significance of $p<0.05, * *$ indicates post hoc test of $p<0.001)$. G: Her 2-NLP treated structures are more likely to contain a mitotic figure than untreated or empty-NLP treated cells ( $n=3$ biological replicates, * indicates post hoc test significance of $p<0.05, * *$ indicates post hoc test of $p<0.001$ ).

Figure 2: Her2-NLPs induce gene expression changes in non-malignant 3D cultures. A: PCA plot for all samples sequence shows that principle component 1 separates biological replicates, and principle component 2 separates Her2-NLP treated from other conditions. B: Genes common to multiple comparisons include BCRYN1 for all NLP treated cells versus untreated samples, and CCL5, CUX2, EBI3, and TGM2 for Her2-NLP treated samples versus either empty-NLP or untreated samples. C: Ingenuity Pathway Analysis network comparing Her2-NLPs to empty NLP discs predicts relationships among several biological families and transcriptional regulators involved in cancer progression and metastasis, including ERK, Akt, P38 MAPK, and STAT3. Genes in red indicate hits found in dataset. Orange coloring indicates predicted activation of biomolecules, and blue indicates predicted inhibition. Deeper color saturation indicates more confidence in predicted regulation. D: Secondary tumor formation is the top activated disease network associated with Her2-NLP treatment compared to untreated samples. E) Organ degeneration is predicted to be inhibited with Her2-NLP treatment compared to untreated samples. Genes in red indicate up-regulated genes found in dataset, whereas genes in purple indicate those that were down-regulated in the dataset. Orange coloring indicates predicted activation and blue indicates predicted inhibition of the biological phenotype. $\log _{2}$ fold change+/-1, $p<0.05$.

Supplementary Figure 1: Her2-NLPs are taken up by cells. A\&B: An untreated cell cultured in 3d IrECM does not show any staining for Her2, whereas C\&D: a treated cell shows abundant Her 2 staining throughout the membrane and cytoplasm, but not nucleus.

Supplementary Figure 2: Gene expression changes associated empty or Her2-NLP treatment. A: Table of differentially expressed genes across all comparisons shows 1 DEG for no-treatment vs. empty NLP treatment. B: List of differentially expressed genes for all comparisons studied. Genes highlighted in yellow are common to both Her2-NLP treated comparisons and in green are common to either NLP treated condition.

Supplementary Figure 3: Her2-NLP treatment activates the regulation of several key biological pathways when compared to untreated cells. Networks identified via Ingenuity Pathways Analysis (IPA) show connections between differentially expressed genes in our dataset and cancer progression and immune regulated biomarkers, such as A: RAS and TNF, B: ERK, TGFB and PI3K, and C: ERK, p38MAPk, and NFkB. Genes in red indicate upregulated genes found in dataset, whereas genes in purple indicate those that were down-regulated in the dataset. Molecules in gray indicate associated connections as predicted through IPA. $\log _{2}$ fold change cutoff $+/-1, p<0.05$.

Supplementary Figure 4: Her2-NLPs induce differentially expressed genes that associate with cancer. A: Her2NLPs link with several cancer-related diseases and biofunctions. B: Eight differentially expressed genes in the Her2NLP vs. empty-NLP comparison demonstrate significant contribution to cancer-related diseases and biofunctions. 
NOTCH3 and TGM2 were present in over $40 \%$ of the predicted cancer-related diseases, with NOTCH3 involved in $47 \%$ and TGM 2 involved in $76 \%$ of hits, respectively. $\log _{2}$ fold change $+/-1, p<0.05$. C: Full list of cancer-related malignancies and corresponding molecular hits when comparing Her2-NLP to empty-NLP discs.

\begin{tabular}{lll}
\hline Acinar Scoring Criteria & Polar (all) & $\begin{array}{l}\text { Somewhat Polar or } \\
\text { Apolar (Any) }\end{array}$ \\
\hline Size & Compact & $>20$ cells \\
\hline Mitotic spindles & Absent & Present \\
$\begin{array}{l}\text { Positioning of ECM } \\
\text { receptors }\end{array}$ & Basal & Lateral or apical \\
\hline $\begin{array}{l}\text { Cell-cell junctions } \\
\text { Colony Border }\end{array}$ & Well organized & Not organized \\
\hline
\end{tabular}

\section{Supplementary Table 1: Acinar Scoring} Criteria.

Supplementary Table 2: Top diseases and biofunctions predicted to be activated (Z-score >2) or inhibited (Zscore<-2) with Her2-NLP treatment compared to untreated cells.

\begin{tabular}{|l|c|c|l|}
\hline Biological Effect & p-value & $\begin{array}{c}\text { Activation z- } \\
\text { score }\end{array}$ & Molecules \\
\hline Secondary tumor & $2.91 \mathrm{E}-06$ & 2.186 & $\begin{array}{l}\text { CCL5, COL4A2, CPA4, CUX2, EBI3, FN1, IL24, NCF2, NCOR2, } \\
\text { NTN4, SERPINA1, TGM2 }\end{array}$ \\
\hline $\begin{array}{l}\text { Synthesis of reactive oxygen } \\
\text { species }\end{array}$ & $9.96 \mathrm{E}-05$ & 2.382 & CCL5,FN1,IL24,IL32,NCF2,SERPINA1,TGM2 \\
\hline $\begin{array}{l}\text { Production of reactive oxygen } \\
\text { species }\end{array}$ & 0.000156 & 2.189 & CCL5,FN1,IL24,IL32,NCF2,TGM2 \\
\hline Organ Degeneration & 0.000238 & -2.219 & ABCG1, CA2, HSD17B2, HSPG2, MTSS1, PITPNM2, SLC7A8 \\
\hline Proliferation of blood cells & 0.000823 & 2.426 & ABCG1, CCL5, EBI3, FN1, IL24, IL32, NCOR2, SERPINA1 \\
\hline Proliferation of lymphocytes & 0.00144 & 2.23 & ABCG1, CCL5, EBI3, FN1, IL24, NCOR2, SERPINA1 \\
\hline Formation of cellular protrusions & 0.00692 & 2.429 & CCL5, CUX2,FN1,MTSS1,NCF2,SEMA6A,SHANK3 \\
\hline
\end{tabular}


bioRxiv preprint doi: https://doi.org/10.1101/2020.08.25.265512; this version posted August 25, 2020. The copyright holder for this preprint (which was not certified by peer review) is the author/funder, who has granted bioRxiv a license to display the preprint in perpetuity. It is made available under aCC-BY-NC-ND 4.0 International license.

Supplementary Table 3: Upstream Factor analysis reveals potential regulators responding to Her2 treatment. Upstream regulators include mediators of inflammation such as LPS, TNF, IFNgamma, Fluticasone Propionate, tetradecanoylphorbol acetate, and/or dexamethasone.

\begin{tabular}{|c|c|c|c|c|}
\hline Regulator & $\begin{array}{l}\text { p-value of } \\
\text { Her2 v. No }\end{array}$ & $\begin{array}{l}\text { p-Value Her2 v. } \\
\text { Empty }\end{array}$ & Target Molecules Her2 v. No & $\begin{array}{c}\text { Target Molecules in Dataset Her2 } \\
\text { v. Empty }\end{array}$ \\
\hline \multirow{2}{*}{ lipopolysaccharide } & & & ABCG1,CCL5,COL4A2,CUX2,EBI3,FN1,IL24,IL32,K & CCL5,CUX2,EB|3,NOTCH3,SYNPO,T \\
\hline & $5.63 \mathrm{E}-06$ & $1.28 \mathrm{E}-05$ & YNU,MTSS1,NCF2,SERPINA1,TGM2 & GM2 \\
\hline fluticasone propionate & 7.39E-06 & $2.55 \mathrm{E}-05$ & ABCG1,CCL5,EBI3,SERPINA1,TGM2 & CCL5,EBI3,TGM2 \\
\hline CCR2 & $9.52 \mathrm{E}-07$ & $7.21 \mathrm{E}-04$ & CCL5,COL4A2,FN1,HSPG2,TGM2 & CCL5,TGM2 \\
\hline NFkB (complex) & 4.03E-08 & $2.12 \mathrm{E}-02$ & $\begin{array}{l}\text { ABCG1,CCL5,FN1,IL24,IL32,KYNU,MTSS1,NCF2,S } \\
\text { LC2A6,TGM2 }\end{array}$ & CCL5,TGM2 \\
\hline albuterol & $2.18 \mathrm{E} 04$ & $1.04 \mathrm{E} 05$ & $\mathrm{CCL5}, \mathrm{EBI} 3$ & $\mathrm{CCL} 5, \mathrm{EBI} 3$ \\
\hline \multirow[b]{2}{*}{ TNF } & & & BCYRN1,CA2,CCL5,EBI3,FN1,HSPG2,IL24,IL32,KY & \\
\hline & 2.85E-06 & 2.75E-03 & NU,NCF2,NCOR2,SLC7A8,TGM2 & CCL5,EBI3,SYNPO,TGM2 \\
\hline PS-1145 & $4.08 \mathrm{E}-04$ & $1.95 \mathrm{E}-05$ & CCL5,EBI3 & CCL5,EBI3 \\
\hline \multirow{2}{*}{ IFNG } & & & BCYRN1,CCL5,CYP24A1,EBI3,FN1,HSPG2,IL32,KY & \\
\hline & $9.08 \mathrm{E}-06$ & $1.05 \mathrm{E}-03$ & NU,MTSS1,NCF2,SERPINA1 & CCL5,EBI3,GLIS1,NOTCH3 \\
\hline IL10RA & $8.60 \mathrm{E}-06$ & $5.24 \mathrm{E}-03$ & $\mathrm{ABCG1,CA2}{ }_{i} \mathrm{CCL} 5, \mathrm{FN} 1, \mathrm{SLC} 2 \mathrm{~A} 6, \mathrm{TGM} 2$ & CCL5,TGM2 \\
\hline E. coli lipopolysaccharide & 2.17E-04 & 3.05E-04 & CCL5,EBI3,IL32 & CCL5,EBI3 \\
\hline CD4OLG & $3.90[-04$ & $3.07[-04$ & CA2,CCL5, [BI3,IL24,TGM2 & CCL5,[BI3,TGM2 \\
\hline \multirow{2}{*}{\begin{tabular}{|l} 
OSM \\
cyanidin 3-O-glucoside
\end{tabular}} & 2.31E-04 & 8.37E-04 & ABCG1,CCL5,FN1,IL32,SERPINA1,SLC7A8 & CCL5,NOTCH3,ZNF467 \\
\hline & $6.76 \mathrm{E}-05$ & $2.89 \mathrm{E}-03$ & ABCG1,CCL5 & CCL5 \\
\hline \multirow{2}{*}{ HRAS } & & & CCL5,CYP24A1,FN1,IL24,LBH,NCOR2,SPSB4,TGM & \\
\hline & $8.59 \mathrm{~F}-06$ & $2.33 \mathrm{~F}-02$ & ) & (C.I. T,TMM) \\
\hline IL12A & $2.41 \mathrm{E}-03$ & 1.17E-04 & CCL5,EBI3 & CCL5,EBI3 \\
\hline IL1B & 1.10E-04 & $4.00 \mathrm{E}-03$ & BCYRN1,CCL5,EBI3,FN1,HSPG2,IL24,IL32,TGM2 & CCL5,EBI3,TGM2 \\
\hline BAX & $3.18 \mathrm{E}-03$ & $1.56 \mathrm{E}-04$ & CCL5,TGM2 & CCL5,TGM2 \\
\hline beta-estradiol & $1.41 \mathrm{E}-05$ & 3.63E-02 & $\begin{array}{l}\text { ADM2,BCYRN1,CA2,CCL5,CYP24A1,FN1,HSD17B } \\
\text { 2.IL24,KYNU,NCF2,PITPNM2,SERPINA1,SLC7A8 }\end{array}$ & CCL5, NOTCH3 SYNPO \\
\hline \multirow{2}{*}{ tetradecanoylphorbol acetate } & & & CA2,CCL5,CYP24A1,EBI3,HSD17B2,IL24,IL32,LBH, & \\
\hline & 7.55E-05 & 7.19E-03 & SERPINA1 & CCL5,EBI3,GLIS1 \\
\hline eflornithine & $3.70 \mathrm{E}-05$ & 1.47E-02 & FN1,IL32,TGM2 & TGM2 \\
\hline PRPF4 & $1.58 \mathrm{E}-03$ & $3.61 \mathrm{E}-04$ & CCL5 & CCL5 \\
\hline TONSL & $1.58 \mathrm{E}-03$ & $3.61 \mathrm{E}-04$ & CCL5 & CCL5 \\
\hline anthraquinone & $1.58 \mathrm{E}-03$ & $3.61 \mathrm{E}-04$ & CCL5 & CCL5 \\
\hline STAR & $1.59 \mathrm{E}-04$ & $4.33 \mathrm{E}-03$ & ABCG1,CCL5 & CCL5 \\
\hline VitaminD3-VDR-RXR & $5.25 \mathrm{E}-05$ & $1.65 \mathrm{E}-02$ & CCL5,CYP2^^1,HSD17B2 & CCL5 \\
\hline LDLR & 3.64E-04 & $2.55 \mathrm{E}-03$ & ABCG1,CCL5,EBI3,HSPG2 & CCL5,EBI3 \\
\hline PRKAA2 & $2.78 \mathrm{E}-05$ & $3.94 \mathrm{E}-02$ & CA2,FN1,NCF2,TGM2 & TGM2 \\
\hline BCL6 & $4.32 \mathrm{E}-04$ & $2.79 \mathrm{E}-03$ & CUX2,EBI3,IL24,LBH & CUX2,EBI3 \\
\hline CAPN3 & $2.52 \mathrm{E}-04$ & $5.41 \mathrm{E}-03$ & CCL5,NCF2 & CCL5 \\
\hline N-ethyl-N-nitrosourea & $2.52 \mathrm{E}-04$ & $5.41 \mathrm{E}-03$ & SERPINA1,TGM2 & TGM2 \\
\hline SIRT1 & 4.03E-03 & $3.42 \mathrm{E}-04$ & ABCG1,CCL5,EBI3,FN1 & CCL5,EBI3,SYNPO \\
\hline phenytoin & 2.87E-04 & $5.77 \mathrm{E}-03$ & CCL5,FN1 & CCL5 \\
\hline PELP1 & 4.29E-05 & 4.40E-02 & HSD17B2,KYNU,NCOR2,TGM2 & TGM2 \\
\hline Immunoglobulin & 8.17E-05 & $2.35 \mathrm{E}-02$ & CCL5,EBI3,FN1,IL24,IL32,NCF2,SAMD11 & CCL5,EBI3 \\
\hline \multirow{2}{*}{ dexamethasone } & & & CA2,CCL5,COL4A2,CPA4,CYP24A1,FN1,IL32,NCO & \\
\hline & $4.28 \mathrm{E}-04$ & $5.06 \mathrm{E}-03$ & R2,NTN4,SERPINA1,TGM2 & $\mathrm{CCL5}, \mathrm{NOTCH} 3, \mathrm{TGM} 2, \mathrm{ZNF} 467$ \\
\hline ARL16 & $3.16 \mathrm{E}-03$ & $7.23 \mathrm{E}-04$ & CCL5 & CCL5 \\
\hline DCTN4 & $3.16 \mathrm{E}-03$ & $7.23 \mathrm{E}-04$ & CCL5 & CCL5 \\
\hline \multirow{2}{*}{$\begin{array}{l}\text { poly-L-glutamic acid-peptoid } 1 \\
\text { conjugate QM56 }\end{array}$} & & & & \\
\hline & $3.16 \mathrm{E}-03$ & 7.23E-04 & CCL5 & CCL5 \\
\hline JAK2 & $1.80 \mathrm{E}-03$ & $1.29 \mathrm{E}-03$ & CCL5,FN1,TGM2 & CCL5,TGM2 \\
\hline Pam3-Cys-Ser-Lys4 & $1.90 \mathrm{E}-03$ & $1.34 \mathrm{E}-03$ & CCL5,EBI3,IL32 & CCL5,EBI3 \\
\hline E. coli B5 lipopolysaccharide & 7.42E-04 & $3.70 \mathrm{E}-03$ & CCL5,IL32,NCF2,TGM2 & CCL5,TGM2 \\
\hline RAS & $5.81 \mathrm{E}-05$ & 4.74E-02 & CYP24A1,FN1,IL24,TGM2 & TGM2 \\
\hline TRAF2 & $7.63 \mathrm{~F}-0.3$ & $3.83 \mathrm{~F}-04$ & C.C. 5,FBI3 & C.CI 5,FBI.3 \\
\hline SB203580 & $6.15 \mathrm{E}-03$ & $4.86 \mathrm{E}-04$ & CCL5,EBI3,FN1,TGM2 & CCL5,EBI3,TGM2 \\
\hline HDAC1 & $8.89 \mathrm{E}-04$ & 4.07E-03 & CCL5,FN1,IL24,TGM2 & CCL5,TGM2 \\
\hline $\begin{array}{l}\text { Salmonella enterica serotype } \\
\text { abortus equi } \\
\text { lipopolysaccharide }\end{array}$ & $2.39 \mathrm{E}-03$ & $1.57 \mathrm{E}-03$ & CCL5,EBI3,IL32 & CCL5,EBI3 \\
\hline GNAI3 & $5.01 \mathrm{E}-04$ & $7.57 \mathrm{E}-03$ & CCL5,IL24 & CCL5 \\
\hline ADORA3 & $5.01 \mathrm{E}-04$ & $7.57 \mathrm{E}-03$ & CCL5,IL24 & CCL5 \\
\hline \multirow{2}{*}{ CTNNB1 } & & & COL4A2,CYP24A1,FN1,HSD17B2,LBH,SEMA6A & \\
\hline & 9.09E-05 & 4.30E-02 & RPINA1,TGM2 & NOTCH3,TGM2 \\
\hline
\end{tabular}


bioRxiv preprint doi: https://doi.org/10.1101/2020.08.25.265512; this version posted August 25, 2020. The copyright holder for this preprint (which plasmid

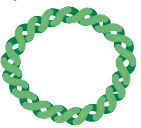
plasmid availablelushirraCC-BY-NC-ND 4.0 International license.<smiles>C=C(C)C1CCCCC1</smiles>

Cell-free lysate

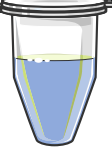

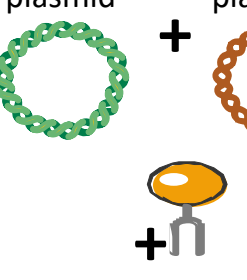

Cell-free lysate

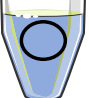

Empty-NLP

HER2-NLP

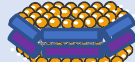

88
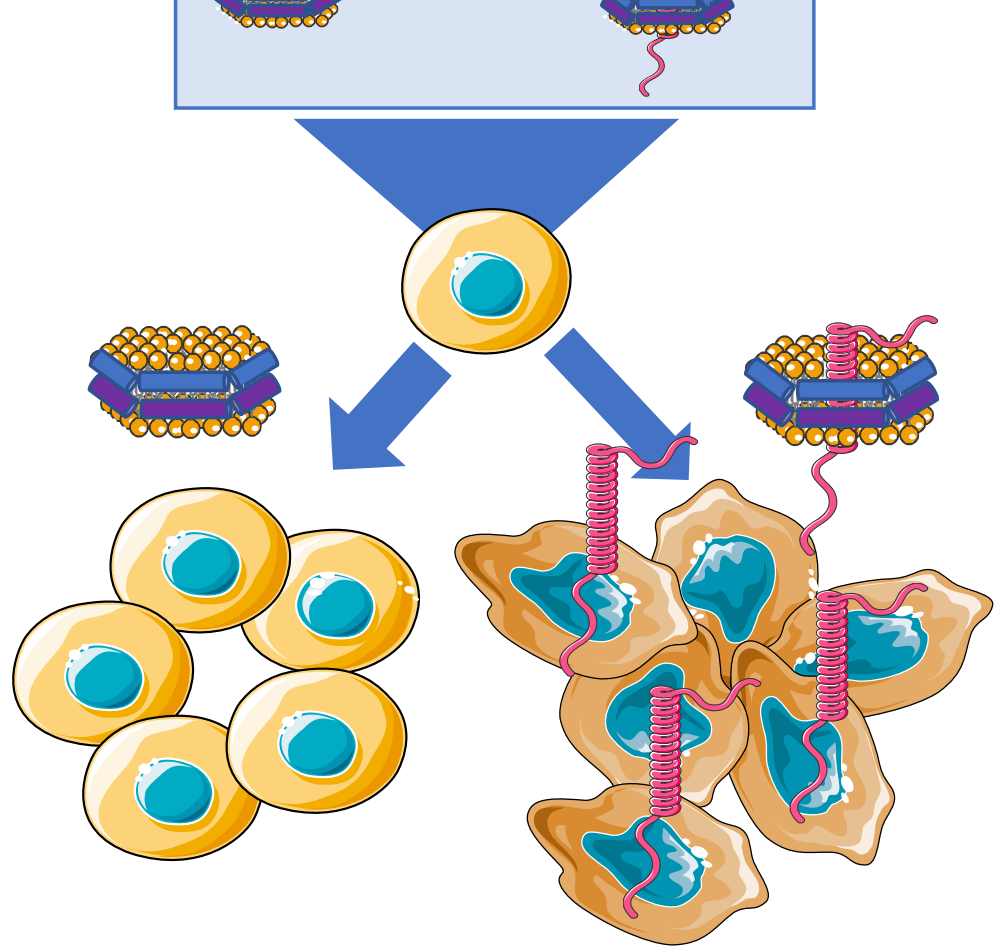


\section{A. Non-malignant}

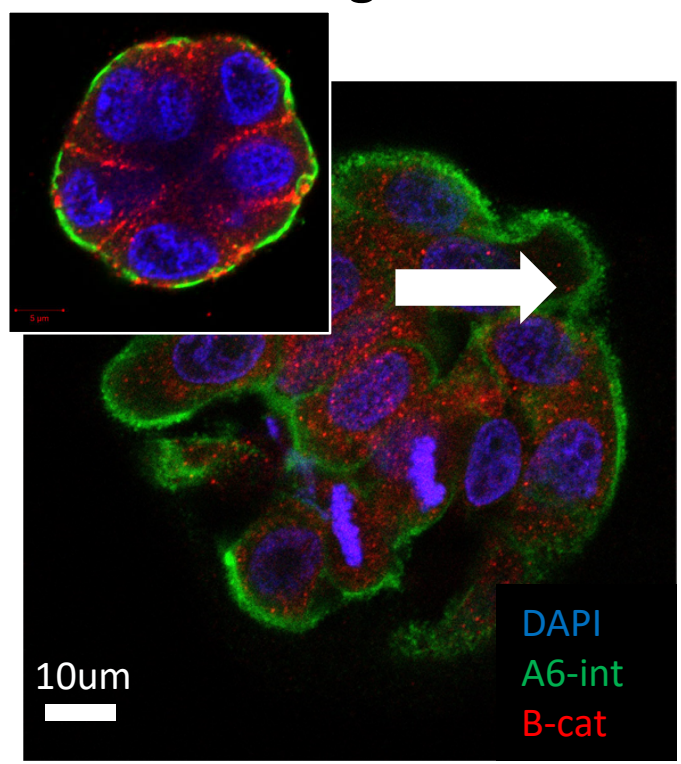

B. Malignant BC

F.
F. Her2-NLPs but not empty or + inhib. Treated non-malignant cells disorganize
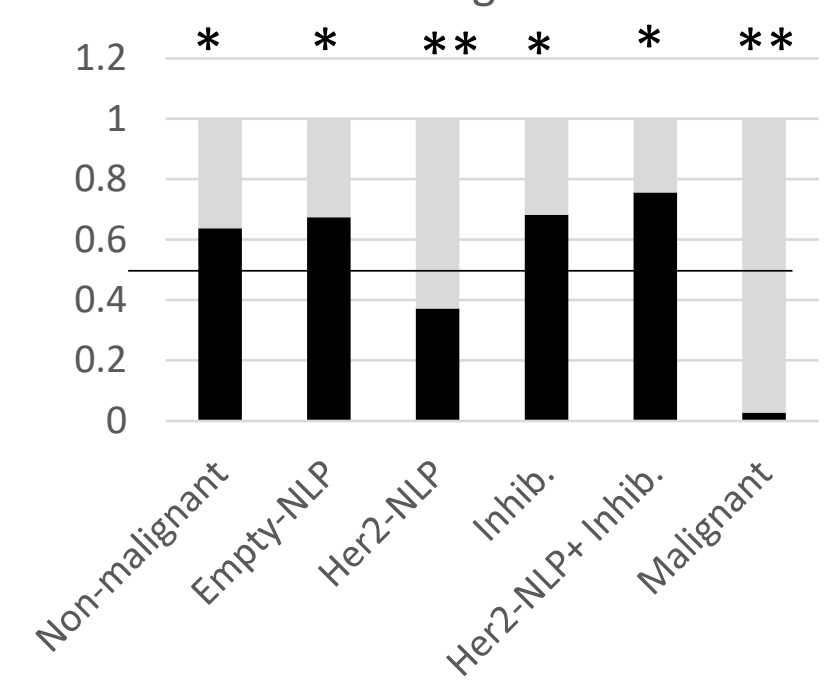

Polar Partially or Apolar

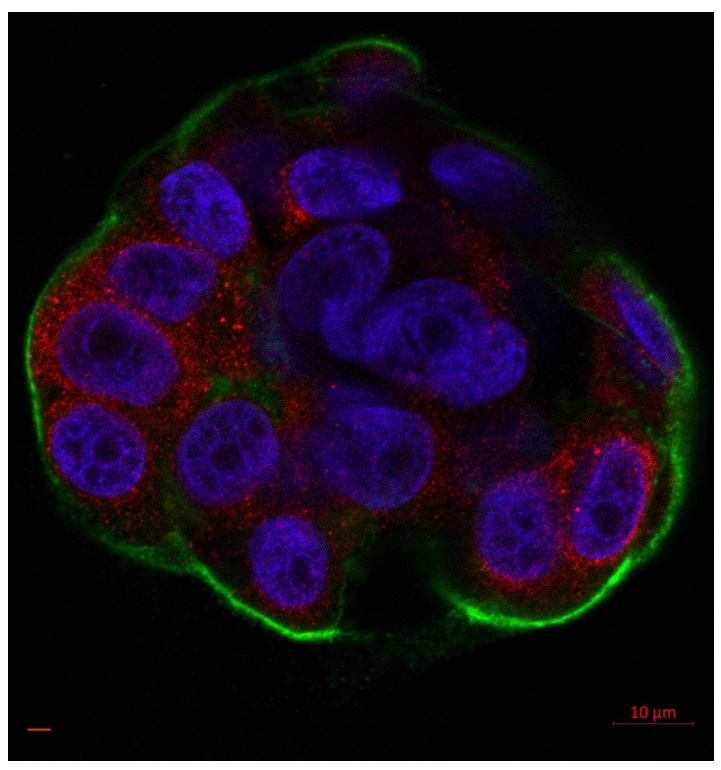

C. + Her2-NLP

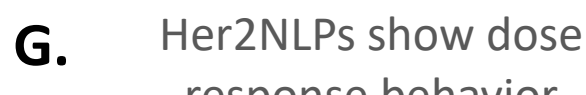
response behavior

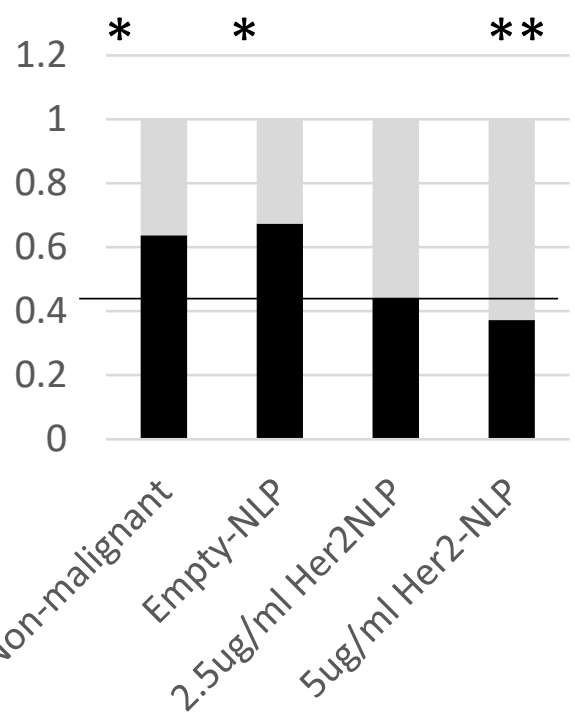

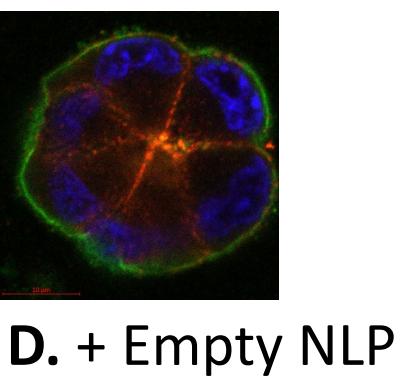

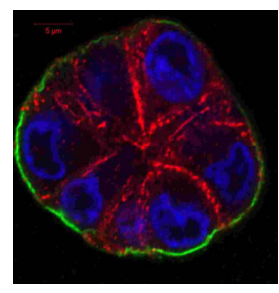

E. + Her2-NLP + Inhib.
H.

Her2-NLPs and mitosis

$* \quad * *$

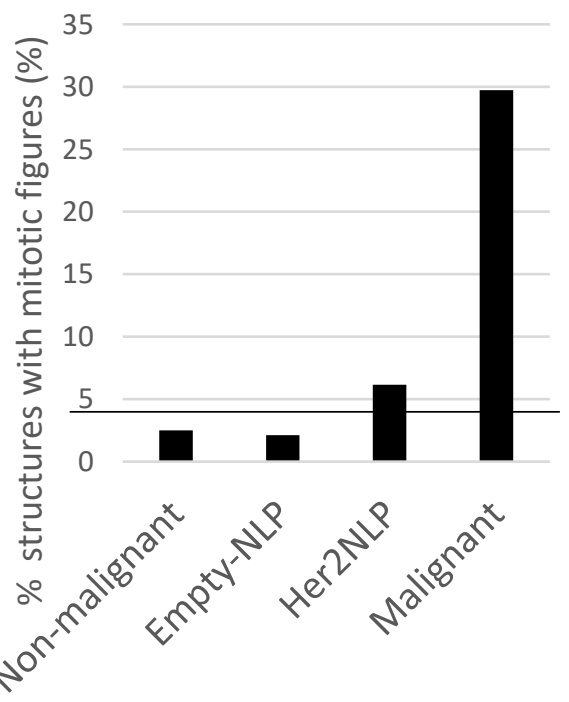


